\title{
Justicia y memoria en la acción de las asociaciones de víctimas de la dictadura en Argentina (1983-2000)
}

por

Nadia Tahir ${ }^{1}$

Université de Caen-Basse Normandie

Entre 1976 y 1983, Argentina vivió la más sangrienta dictadura de su historia. El método represivo más emblemático de la acción de las juntas militares al poder fue la desaparición forzada de personas. Tras el final de la dictadura, las asociaciones de familiares y la de ex detenidos desaparecidos se implicaron en la lucha por la "Verdad, justicia y memoria» ligada a la gestión del pasado dictatorial en democracia. Este trabajo se propone volver sobre los discursos y la acción de estas agrupaciones durante los mandatos de los presidentes Raúl Alfonsín (1983-1989) y Carlos Menem (1989-1999). Se trata de ver cómo han evolucionado, pasando de un periodo en el que sus reivindicaciones están en un primer plano de la esfera pública y de la agenda gubernamental a convertirse en oposición en un tiempo de políticas llamadas de «reconciliación nacional». Veremos cómo los actores se centran en el mantenimiento de sus reclamos y en qué medida la nueva coyuntura permite la emergencia de nuevos actores. Se trata de analizar cómo el conjunto de estos actores se remite a las herramientas judiciales y memoriales para seguir su labor y subrayar las singularidades del caso argentino en la construcción de una memoria colectiva.

Palabras clave: Argentina; pasado dictatorial; asociaciones; justicia; memoria.

${ }^{1}$ Este artículo proviene de una tesis doctoral presentada el 7 de diciembre de 2011 en la Universidad Paris-Sorbonne (Paris IV), bajo la dirección del profesor Miguel Rodríguez: «Les assotiations de victimes de la dictature: politiques des droits de l'homme et devoir de mémoire en Argentine (1976-2007)» [«Las asociaciones de víctimas de la dictadura: políticas de derechos humanos y deber de memoria en Argentina (1976-2007)»]. Agradezco a Claudia Feld, Sophie Burdet, Vanessa Silva y Ariadna Barroso la pertinencia de sus comentarios a una primera versión de este texto. También quiero agradecer a Mónica Quijada su ayuda y su interés por mi trabajo y a Miguel Rodríguez su apoyo constante. 
La desaparición forzada de personas es en la actualidad un crimen contra la humanidad reconocido por instancias nacionales e internacionales ${ }^{2}$. En Argentina, fue el principal instrumento de la represión ejercida por las Fuerzas Armadas durante la última dictadura militar que conoció el país entre 1976 y 1983. Numerosas asociaciones de derechos humanos actuaron para denunciar los crímenes cometidos por el régimen militar ${ }^{3}$. Dentro de estas agrupaciones, algunas se distinguieron al estar compuestas por familiares de personas desaparecidas. Durante la dictadura, el conjunto de estas asociaciones ${ }^{4}$ no dejó de interpelar a las comunidades nacionales e internacionales para que se reconociera lo que en aquel momento no era un crimen contra la humanidad: la desaparición forzada de personas.

Con la restauración de las instituciones constitucionales en 1983, la acción de estas asociaciones sigue adelante y, hoy en día, a pesar de que hayan transcurrido más de veinticinco años, continúan estando muy presentes en el espacio social argentino ${ }^{5}$. Tras el mandato de Néstor Kirchner (2003-2007), las cuestiones ligadas a la desaparición forzada de personas y a la represión dictatorial son evocadas ampliamente en la prensa nacional argentina y en la prensa internacional: inauguración de museos de la memoria, placas conmemorativas, juicios

2 Para más detalles, ver la Convención Internacional sobre la desaparición forzada de personas en la página web del Alto Comisionado por los Derechos Humanos de la Organización de las Naciones Unidas: http://www.ohchr.org/Documents/Publications/FactSheet6Rev3 sp.pdf. Conviene señalar que el término «Desaparición forzada de personas» corresponde a la denominación jurídica del crimen. En otros ámbitos y cuando un actor no se refiere al crimen en términos jurídicos, se habla de «desaparición».

3 Para más detalles sobre los primeros años del movimiento de derechos humanos, Leis, 1989.

${ }^{4}$ Se trata de las asociaciones de familiares de detenidos-desaparecidos (La Asociación Madres de Plaza de Mayo, las Madres de Plaza de Mayo Línea Fundadora, las Abuelas de la Plaza de Mayo, Familiares de desaparecidos y detenidos por razones políticas, H.I.J.O.S. Hijos por la Identidad y la Justicia contra el Olvido y el Silencio) y de una asociación de sobrevivientes de los centros clandestinos de detención (la Asociación de exdetenidos desaparecidos), ubicada en Buenos Aires. Para referirnos al conjunto de estas asociaciones hablaremos de «las asociaciones de víctimas» en la medida en que la categoría de «víctima» las agrupa. Aunque aquí se evoquen en conjunto, esto no significa que no haya conflictos y tensiones entre ellas. Asimismo, cabría analizar con detalle la noción de «víctima» en este caso. Sin embargo, teniendo en cuenta la óptica de este artículo, no podremos detenernos en tratar con detalle estos temas.

5 No son las únicas asociaciones de víctimas de la dictadura, ya que otras asociaciones de carácter regional se fundaron desde entonces. Hemos elegido trabajar sobre estas asociaciones porque son las que tienen más visibilidad mediática, al haber nacido en la provincia de Buenos Aires y al actuar en la zona de la Capital Federal, Buenos Aires. 
contra represores ${ }^{6}$. Sin embargo, esto no siempre ha sido así. Como bien lo señala Enzo Traverso, el caso argentino se distingue debido a varios elementos. Supone la presencia de una «memoria de los crímenes de la dictadura militar» ya durante el régimen y se refiere a los colectivos objeto de nuestro artículo. Pero subraya también las singularidades de la desaparición forzada de personas que conlleva una «prolongación» de la «fase de duelo y de aflicción». Lo cual hizo que no hubiese «olvido». En este marco se sitúa la acción de las asociaciones de víctimas que, sin embargo, como bien lo señala Traverso, se ubican también dentro de las singularidades de la «transición hacia la democracia», que fue «sin ruptura radical, sin verdadera depuración de las instituciones militares, con algunos juicios seguidos por leyes de amnistía que llevaron a la impunidad de los represores» ${ }^{7}$. Para poder estudiar la articulación entre «la memoria» y la «justicia», en la acción y los discursos de las asociaciones de víctimas, conviene, entonces, tener en cuenta estos distintos marcos.

Por otra parte, Paul Ricoeur en su ensayo La memoria, la historia, el olvido plantea que:

La justicia es la que, sacando de los recuerdos traumatizantes su valor ejemplar, convierte a la memoria en proyecto, y es ese mismo proyecto de justicia el que da al deber de memoria la forma del futuro y del imperativo. Podemos entonces sugerir que el deber de memoria, como imperativo de justicia, se proyecta como un tercer término en el punto de concurrencia entre el trabajo de duelo y la labor de memoria ${ }^{8}$.

Partiendo de esta reflexión, veremos en qué medida las acciones y los discursos de las asociaciones han contribuido al mantenimiento de las cuestiones ligadas al pasado dictatorial en Argentina. Aunque el «deber de memoria» se impuso desde un principio en su lucha, huelga decir que provenía de la necesidad de un reclamo de justicia. Así es como, cuando ya no se podía obtener justicia, las asociaciones de víctimas se orientaron hacia una siguiente etapa, «la labor de memoria».

En este trabajo, quisiéramos volver a los años que precedieron a los cambios ligados a la gestión de Néstor Kirchner y, sobre todo, a los dos mandatos del presidente Carlos Menem (1989-1999). Se trata de entender cómo se han articulado los reclamos por la justicia y cuál ha sido la «labor de memoria» emprendida por las asociaciones de víctimas de la dictadura, durante una

${ }^{6}$ El mandato de Néstor Kirchner ha respondido a un gran número de exigencias de las asociaciones que son objeto de este artículo: anulación de las leyes de amnistía, «recuperación» de los centros clandestinos de detención, que se convierten hoy en museos y espacios por la memoria.

7 Traverso, 2005: 52. Traducción de la autora.

8 Ricoeur, 2000: 107. Traducción de la autora. 
época en la cual las cuestiones ligadas al pasado dictatorial no tienen tanto protagonismo político y social. A pesar de ser relegadas a un segundo plano en la esfera pública argentina, ¿cómo lograron estas asociaciones mantenerse en el espacio social argentino?

Para mayor claridad, este artículo seguirá un orden de reflexión cronológico. Una primera etapa empieza en el año 1983 y termina en torno al año 1990, cuando el reclamo por la justicia es posible. La segunda fase corresponde al periodo entre los años 1990 y 1995 y abarca lo que se suele calificar como la época de «vacío jurídico». Finalmente, la tercera etapa de este trabajo se iniciará en el año 1995 y se cerrará cerca del año 2000, cubriendo el periodo de tiempo en que las luchas de las asociaciones de víctimas por la justicia y «la memoria» se materializan en el nacimiento de la asociación H.I.J.O.S.

\section{«Juicio y CAStigo A todos los CUlPables» (1983-1990)}

El 10 de diciembre de 1983 Raúl Alfonsín asume el poder como primer presidente constitucional al terminar la dictadura. Candidato de la Unión Cívica Radical (UCR), Alfonsín también fue miembro de una de las asociaciones de derechos humanos más importantes, la Asamblea Permanente por los Derechos Humanos (APDH). Cuando accede al poder, conoce bien los reclamos de las asociaciones de víctimas. En este nuevo contexto político, las familias de personas desaparecidas y las asociaciones que se crearon durante el régimen anterior, Familiares ${ }^{9}$, Madres ${ }^{10}$ y Abuelas ${ }^{11}$ vislumbran la posibilidad de obtener respuestas a las preguntas que rodean la desaparición de sus seres queridos ${ }^{12}$.

9 La asociación de Familiares de desaparecidos y detenidos por razones políticas (Familiares) se fundó en diciembre de 1976. Folleto editado por la asociación de Familiares de detenidos desaparecidos por razones políticas para los 30 años de su existencia en el año 2006: Testimonios de nuestra lucha 1976-2006, Buenos Aires. Entrevista de la autora con IDV, miembro fundador de Familiares, el 21 de diciembre de 2006.

10 La asociación de Madres de Plaza de Mayo nació el 30 de abril de 1977. Sufrió una división en el año 1986, de la que nacieron dos asociaciones muy distintas: la Asociación Madres de Plaza de Mayo (MPM) presidida por Hebe de Bonafini y la asociación de las Madres de Plaza de Mayo Línea Fundadora (Madres LF). Para más detalles, véase Gorini, 2006. Gorini, 2008.

11 La asociación de las Abuelas de Plaza de Mayo nació en octubre de 1977. Centra sus esfuerzos en la búsqueda de los niños secuestrados, de padres desaparecidos o nacidos durante el cautiverio de sus madres. A fecha de mayo de 2011, se ha restituido la identidad a 103 hijos de desaparecidos. Véase la página web de la asociación: www.abuelas.org.ar.

12 Hasta se habló de que había desaparecidos en vida. Mellibovsky, 1989 (2006): 154. 
Durante toda su campaña electoral, Raúl Alfonsín desarrolló un discurso centrado en la defensa de los derechos humanos ${ }^{13}$. Pocos días después de asumir la presidencia, Alfonsín publica dos decretos que instauran los dos pilares de su llamada política de derechos humanos ${ }^{14}$. Para empezar impulsa la creación de la Comisión Nacional sobre la Desaparición de Personas (CONADEP), cuyos principales objetivos son el de investigar las desapariciones de personas y el de determinar las características de la represión militar en Argentina. En 1984, se publica el informe de la Comisión: Nunca Más ${ }^{15}$. Este documento se convierte en una referencia, y lo sigue siendo, al hablar de la desaparición forzada de personas en Argentina ${ }^{16}$.

El segundo pilar es el juicio a las juntas militares que tendrá lugar entre abril y diciembre de $1985^{17}$. Durante estos meses, los nueve miembros de las tres primeras juntas militares que dirigieron Argentina entre 1976 y 1982 fueron juzgados ${ }^{18}$. Al término del juicio, dos fueron condenados a cadena perpetua, tres a penas de prisión de entre cuatro y diecisiete años y los demás fueron liberados. Como bien señalan Carlos Acuña y Catalina Smulovitz, más allá de la carga simbólica que representó el juzgar a los que ejercieron el poder durante la dictadura, tan poco tiempo después de terminarse esta, lo más significativo durante el juicio fue que:

La lógica jurídica, expuesta públicamente, tuvo la capacidad de ordenar el pasado, dar verosimilitud y dejar fuera de toda sospecha al relato de los testigos, constituyéndose en un efectivo mecanismo para el juicio histórico y político del régimen dictatorial. [...] El juicio a las Juntas, que en la estrategia gubernamental debía configurar el fin de la «cuestión derechos humanos» terminó reabriendo el tema ${ }^{19}$.

Desde los primeros años del regreso del gobierno constitucional, hay una clara voluntad por parte de los dirigentes de ligar el restablecimiento del Estado de derecho a la vía judicial.

13 Nino, 1997 (2006): 119.

14 No son los únicos elementos de la política llevada a cabo por Raúl Alfonsín, pero son los más emblemáticos. Véase: Pereyra, 2008: 35.

15 CONADEP, 1985. En 2006, se publica de nuevo este informe con un nuevo prólogo: CONADEP, 2006.

16 Crenzel, 2008: 18.

17 Idem.

18 Los militares en cuestión son: Jorge Rafael Videla, Eduardo Massera, Roberto Eduardo Viola, Armando Lambruschini, Orlando Ramón Agosti, Omar Graffigna, Leopoldo Galtieri, Jorge Isaac Anaya, Basilio Lami Dozo.

19 Acuña y Smulovitz, 1995: 58. 
En este contexto, nace una de las consignas más emblemáticas de las asociaciones de víctimas y de defensa de los derechos humanos: «Juicio y castigo a todos los culpables». Estas agrupaciones tratan de responder a la acción del nuevo gobierno. En un principio hasta se podría decir que esta consigna tenía como objetivo apoyar las iniciativas del gobierno a pesar de no estar del todo satisfechos con la CONADEP ${ }^{20}$ o con el juicio a las juntas. En efecto, las asociaciones de víctimas, y algunas asociaciones de derechos humanos, como el Servicio Paz y Justicia (Serpaj), pedían que se creara una comisión bicameral que implicase al Parlamento argentino y a la justicia penal. El objetivo era que las denuncias hechas en la CONADEP tuvieran consecuencias penales directas al pasar de la comisión a los juzgados. Por otra parte, para las asociaciones había que juzgar a todos los miembros de las cuatro juntas militares que dirigieron el país. La última junta quedó fuera del juicio. También planteaban que había que juzgarles por un mayor número de crímenes y no solo por algunos casos, como en los que decidió centrarse la acusación. El gobierno de Raúl Alfonsín rechazó esas opciones, pero, como señalan Acuña y Smulovitz, el juicio a las juntas solo era un comienzo para los familiares. Tras unas condenas que les parecieron decepcionantes, las asociaciones de víctimas incrementaron su ayuda a las personas que querían denunciar en los juzgados la desaparición de un ser querido.

En cuanto a la lucha judicial, conviene referirse a otro elemento evocado por la cita de Acuña y Smulovitz: el papel del juicio a las juntas en el establecimiento de la legitimidad de los testigos. Precisamente entonces, en el ámbito judicial, las declaraciones que los sobrevivientes de los centros clandestinos venían haciendo, incluso durante la dictadura, van a cobrar sentido de «verdad» ${ }^{21}$. La asociación de ex detenidos desaparecidos nace en 1984

20 Crenzel, 2008: 53-103.

21 Para entender la importancia de este juicio en la visión que se tenía de los sobrevivientes, cabe evocar brevemente algunos discursos que circulaban en esta época, y siguen circulando en parte, sobre los sobrevivientes de los centros clandestinos. Primero, seguía muy presente la idea de que si se habían llevado a una persona «por algo sería», algo tendría que haber hecho para que esto pasara. En este sentido se implementó más tarde la «teoría de los demonios», que difundió la idea de que, si efectivamente hubo represión militar, fue para hacer frente al terrorismo - refiriéndose a la guerrilla urbana de izquierda en activo en los años 1970 en Argentina- A esto hay que añadir el hecho de que los sobrevivientes eran sospechosos de haber colaborado con los militares para poder salir de los centros clandestinos de detención. La suma de estos discursos, y de otros, dejó a los sobrevivientes, durante mucho tiempo, al margen de las luchas por la verdad, la justicia y la memoria. Véase: Longoni, Ana, Traiciones, Buenos Aires, Norma, 2007; Vezzetti, Hugo, Pasado y Presente. Guerra, dictadura y sociedad en la Argentina, Buenos Aires, Siglo XXI editores Argentina, 2003; Franco, Marina y Tahir, Nadia, «Associations de victimes, terrorisme d'État et politi- 
durante la preparación del juicio. Los miembros de esta asociación señalan que el primer objetivo era el de reunir testimonios y presentárselos a la fiscalía para que esta seleccionara a los que irían a testificar ante los jueces ${ }^{22}$. $\mathrm{Su}$ nacimiento está entonces sumamente ligado a la batalla judicial vigente en estos primeros años de democracia. Según Inés González Bombal:

La CONADEP apareció como un lugar legítimo de escucha y de registro de testimonios al que pudieron concurrir las víctimas. [...] Por primera vez las víctimas accedieron a los medios de comunicación masivos y se expresaron ${ }^{23}$.

La escena del juicio a las Juntas simbolizó así la instauración de un nuevo contrato social que reconocía la prioridad del derecho y garantizaba la neutralidad del Estado. El Juicio a las Juntas tuvo efectos simbólicos cruciales para el orden político, demostró que las premisas básicas del estado de derecho podían pasar de la enunciación al acto. [...] Si el testimonio es la extinción de la desaparición, el juicio a las Juntas representó el imaginario de la completitud del estado de derecho ${ }^{24}$.

En 1986 se vota la Ley de Punto Final, que da un plazo de sesenta días, tras ser promulgada, para que se inicie una querella. Las personas que no hubiesen sido llamadas a declarar ante la justicia antes de ese plazo ya no podrían ser objeto de querellas. En 1987, tras una serie de presiones militares se vota la Ley de Obediencia Debida, que establece que ya no se pueden entablar juicios a personas que hayan seguido órdenes de sus superiores, como es el caso de los militares durante la última dictadura. Con estas dos leyes, se pone fin a las posibilidades que contemplaban las familias y los exdetenidos desaparecidos de juzgar a los que habían cometido los crímenes de la dictadura $^{25}$. La lucha se torna entonces en una lucha contra las decisiones del gobierno de Raúl Alfonsín. Pocos años después será en contra del gobierno de Carlos Menem, quien indultará ${ }^{26}$ a todas las personas que fueron condenadas durante los primeros años de la democracia, incluidos los excomandantes,

que dans l'Argentine de 1973 à 1987», Vingtième Siècle. Revue d'Histoire, 105 (París, 2010): 185-198.

22 Entrevista de la autora con $\mathrm{OB}$, miembro de la asociación de exdetenidos-desaparecidos, 15 de diciembre de 2006.

23 González Bombal, Inés, «"Nunca más”, el juicio más allá de los estrados», en C. Acuña et al., Juicio, castigos y memorias, Buenos Aires, Nueva Visión, 1995: 204.

24 González Bombal, 1995: 212.

25 Existen dos excepciones que no contemplan estas dos leyes: los niños apropiados, casos de los que se encargan las Abuelas, y el robo de bienes inmobiliarios. Son las únicas querellas y juicios que se llevarán a cabo en los años 1990.

26 En 1989 y en 1990, el presidente Carlos Menem indulta a los miembros de las juntas condenados durante el juicio de 1985 y a todos los militares condenados antes de las leyes de Punto Final y de Obediencia Debida. 
durante el juicio a las juntas. Ahora bien, aunque sea una batalla contra decisiones del poder ejecutivo, siguen siendo reclamos por la justicia y hasta podríamos hablar de reclamos contra la impunidad. Se trata de luchar por la posibilidad de juzgar a los culpables de los crímenes de la dictadura.

Durante la dictadura y el gobierno de Raúl Alfonsín la lucha de las asociaciones de víctimas siempre estuvo ligada a la urgencia: la posibilidad de recuperar a los desaparecidos, de saber qué les pasó, de castigar a los culpables, la necesidad de informar a los ciudadanos argentinos de lo ocurrido. Al estudiar la acción y los discursos de estas asociaciones, uno percibe que en esta época no se trataba de proyectarse a largo plazo, sino de actuar en el presente:

No podemos omitir la condena a aquellos que públicamente han abjurado y deshonrado esa lucha, arrogándose el derecho de asumir en nombre del campo popular una reconciliación con los asesinos, secuestradores, torturadores y expoliadores de nuestro pueblo, negociando en pos de una supuesta pacificación nacional a miles de detenidos, desaparecidos, muertos y exiliados que dieron su vida y su libertad por una sociedad justa y pidiendo perdón para los responsables y ejecutores de la represión más sangrienta de nuestra historia, en lugar de exigir para ellos Juicio y Castigo 27 .

En los primeros años de la democracia, el reclamo por la justicia se conformó dentro de esta lógica, se trataba de obtener juicios, condenas y castigos para todas las personas que habían participado de una forma $u$ otra en la represión dictatorial. Con la instauración de la impunidad desde 1986, y aún más a partir de 1990, las asociaciones se ven ante la necesidad de replantear sus estrategias discursivas y militantes; se encuentran en un momento crítico de su acción y de su existencia. La imposibilidad de continuar la lucha judicial llevó a muchos miembros de las asociaciones a abandonarlas ${ }^{28}$. Asimismo, esta imposibilidad de luchar a nivel judicial y el repliegue de la sociedad hacía sí misma, tras la crisis económica de 1989 y gracias a las políticas económicas y sociales implantadas por Carlos Menem, reducen drásticamente el apoyo recibido por parte de la sociedad civil a estas asociaciones ${ }^{29}$. Al

27 Familiares de desaparecidos y detenidos por razones políticas, «No al indulto», revista de Familiares, Decimos, XII/3 (Buenos Aires, 1989): 1.

28 Entrevista de la autora con AS, Madre de Plaza de Mayo LF, los 22 y 27 de noviembre de 2006. Entrevista de la autora con IDV, miembro de Familiares, el 21 de diciembre de 2006.

29 Novaro, 2006: 250-251. También cabe señalar que ya no se benefician de muchas ayudas internacionales, ya que estas estaban condicionadas al hecho de que las asociaciones lucharan durante una dictadura. Con la instauración de un Estado de derecho, las instituciones o los grupos que les ayudan se dirigieron hacia otras causas, otros países. 
iniciarse los años noventa, las asociaciones de víctimas de la última dictadura dejan la lucha por el reclamo judicial en un segundo plano y centran sus fuerzas en otras reivindicaciones.

\section{LA LUCHA CONTRA LAS POLÍTICAS DEL «PERDÓN» (1990-1995)}

Con la restauración de la democracia, las asociaciones se distancian las unas de las otras. Ya no tienen por qué unirse para oponerse a la dictadura ${ }^{30}$. Las diferencias entre las asociaciones son cada vez más significativas. Cuando Carlos Menem gana las elecciones en 1989, hay cinco asociaciones de víctimas $^{31}$. Divididas sobre numerosos temas ${ }^{32}$, se unen puntualmente de nuevo para oponerse a los indultos presidenciales en 1989 y 1990. La voluntad clara de Carlos Menem de pasar página y de practicar una política llamada del «perdón»" ${ }^{33}$ afecta ampliamente a las asociaciones. Las manifestaciones son multitudinarias e importantes ${ }^{34}$, pero no logran que el gobierno los retire.

A partir de esta época empieza lo que puede calificarse como el periodo de «vacío jurídico». Entre 1987 y 2005 las asociaciones no podrán iniciar ningún tipo de proceso jurídico ${ }^{35}$. La causa de los desaparecidos pasa a un segundo plano. Al estar menos presentes en la esfera pública argentina, las asociaciones de víctimas van a tener que desarrollar otras facetas de su lucha. Así es como el «deber de memoria» pasa a protagonizar sus labores y sus discursos diarios. Se trata de mantener a los desaparecidos en la esfera pública para que la gente que luchó junto a estas asociaciones en los años 1980 no piense que el tema está cerrado. Esta tarea no será fácil, entre otras cosas, porque llega otro contingente de personas al que hay que concienciar sobre estos temas: los argentinos que no han vivido la dictadura, muy jóvenes todavía en los primeros años de la democracia.

30 Tahir, 2009: 233-249.

31 Como ya comentamos antes, en 1986 las Madres de Plaza de Mayo se dividen en dos asociaciones, la Asociación de Madres de Plaza de Mayo (MPM) y las Madres de Plaza de Mayo Línea Fundadora (Madres LF).

32 Dentro de esta reflexión, no podemos volver en detalle sobre las diferencias entre las asociaciones. La MPM es la única asociación que no volvió a unirse con las otras. En cuanto al resto de las asociaciones, las de familiares y la de sobrevivientes de los centros clandestinos de detención se reúnen con cierta regularidad, aunque actúen cada una por su cuenta.

33 Para más detalles sobre las llamadas políticas del «perdón»o de la «reconciliación», véase Lefranc, 2002.

34 Jelin, 2005: 545.

35 Con excepción de los casos evocados en una nota a pie de página anteriormente. 
A lo largo de los años 1990, aunque las cuestiones ligadas a los desaparecidos siguen estando presentes, las asociaciones de víctimas luchan contra los problemas del momento en Argentina. La Asociación Madres de Plaza de Mayo (MPM) milita junto a movimientos obreros, de estudiantes y con partidos políticos de la extrema izquierda. Reivindica las luchas obreras y estudiantiles de los años 1960 y 1970, insistiendo en el hecho de que la lucha de sus hijos desaparecidos ${ }^{36}$ continúa. Los discursos de las otras asociaciones son a menudo cercanos a los de las MPM. Se ven de nuevo referencias a las mismas militancias y hasta se repiten fórmulas, sobre todo cuando se trata de establecer que sus hijos han dado la vida por la libertad y por un gran número de derechos de los cuales se beneficia el pueblo ${ }^{37}$. Pero no militan con ningún sector en particular. Se unen principalmente a asociaciones de derechos humanos. Si coinciden con partidos políticos o sindicatos es exclusivamente durante grandes manifestaciones, como la conmemoración del golpe de Estado, cada 24 de marzo, o la Marcha de la Resistencia. Esta marcha, que dura veinticuatro horas en la plaza de Mayo, tuvo lugar por primera vez en 1981 a iniciativa de las Madres de la plaza de Mayo. Suele ocurrir en torno al 10 de diciembre, día para la defensa de los derechos humanos, y reúne a un gran número de colectivos. Entonces, las modalidades de lucha no son siempre las mismas, sin embargo, los discursos se asemejan. Lo más significativo es el uso de una misma palabra por parte de todas las agrupaciones: «la memoria». Se trata de constituir un discurso en torno a los desaparecidos y a los crímenes de la dictadura. Hay que dar a entender a las nuevas generaciones que las políticas llamadas del «perdón» de Carlos Menem tienen consecuencias en el presente, y también para el futuro. Así es como para las MPM, la evocación recurrente de los desaparecidos no se hace en el recuerdo, sino en la producción ${ }^{38}$, en una continuidad: «Las Madres tenemos memoria fértil. A la vez que recordamos, construimos con los jóvenes» ${ }^{39}$. Madres LF,

36 «Los 30.000 [...] siguen creando, viviendo y luchando con el pueblo», Editorial, Revista de las Madres de Plaza de Mayo, VIII/86 (Buenos Aires, 1992): 1.

37 «Lucharon por la vida y la libertad, el derecho al trabajo, salario justo, vivienda digna, salud y educación para todos. Contra la dependencia imperialista, por un proyecto de liberación, hoy están desaparecidos», Editorial, Decimos, Revista de Familiares, II/4 (Buenos Aires, 1998): 1.

38 Para las MPM se trata de actuar ayudando a los sectores con los que manifiestan. Esto se traducirá a finales de los años noventa y principios de los 2000 con la creación de una Universidad de las Madres. En este espacio se pretende formar a una nueva generación de jóvenes argentinos en acuerdo con los ideales de los desaparecidos. Véase: http://www.madres. org/univupmpm/univumpm.asp.

39 Editorial, «Tenemos una memoria fértil», Revista de las Madres de Plaza de Mayo, XIV/150 (Buenos Aires, 1998): 3. 
Familiares y Abuelas utilizan discursos parecidos. Por ejemplo, el 23 de mayo de 1994 convocan a la población argentina para que participe en una emisora de radio: Radio Memoria. En el llamamiento para participar en esta emisora, escriben:

En los 70 el terrorismo de Estado utiliza esta práctica [la desaparición forzada de personas] de forma masiva y sistemática en el Cono Sur y luego, en toda Latinoamérica, para sofocar las luchas de liberación surgidas contra la injusticia social de un sistema social violador de todos los derechos humanos: la vida, la libertad, el trabajo, el sueldo justo, la educación, la salud, la vivienda ${ }^{40}$.

Es interesante ver que todo por lo que luchaban los desaparecidos corresponde a todo por lo que luchan numerosos sectores sociales en 199441. Ya no se trata de aportar soluciones a los errores del pasado reclamando justicia, o hasta de procurar que los hechos no se repitan. Al luchar por «la memoria», se persigue la lucha de los desaparecidos, que ya no pertenecen a un pasado más o menos lejano, sino, al contrario, a una actualidad muy vigente. «La memoria» se convierte aquí en el principal vector de la continuidad de la lucha, no solo forma parte de la retórica de oposición a las políticas llamadas del «perdón», sino que también se opone a las políticas económicas y sociales de Carlos Menem. Podemos pensar que esta nueva orientación de las asociaciones de víctimas ha tenido sus repercusiones, ya que resisten a los discursos difundidos y alimentados por los sectores que apoyan las llamadas políticas del «perdón». No obstante, habrá que esperar al año 1995 para que estos nuevos discursos tengan repercusiones significativas.

Al mismo tiempo, cabe señalar que algunas asociaciones utilizan formas de acción propias de las luchas memoriales, como pueden ser las conmemoraciones y los homenajes ${ }^{42}$. Las MPM se oponen categóricamente a estas modalidades $^{43}$, pero las otras asociaciones las reivindican y multiplican las actividades en este sentido. Estas iniciativas no se benefician de una cobertura mediática significativa ${ }^{44}$, pero son una oportunidad de encuentro puntual

40 Llamamiento para participar en Radio Memoria para la semana internacional del detenido-desaparecido, 23 de mayo de 1994.

41 Pereyra, 2008: 57-88.

42 Acerca de las conmemoraciones y homenajes, véase: Nora, 1997. Jelin, 2002: 245250.

43 Folleto Nuestras consignas, publicado por la Asociación de Madres de Plaza de Mayo (MPM), verano de 2008, «Rechazamos los homenajes póstumos».

44 El único periódico que hace referencia con mucha regularidad a estas manifestaciones es Página 12 (fundado en 1987 y muy comprometido en las luchas ligadas a los desaparecidos). 
para los miembros de estas asociaciones. Suelen estar organizadas por las propias asociaciones o por antiguos alumnos de una escuela o empleados de una misma empresa que desean poner una placa o rendir un homenaje allí donde los desaparecidos trabajaron, vivieron o estudiaron. Estas acciones se distinguen de los discursos que acabamos de evocar en la medida en que no son apoyadas por el conjunto de las asociaciones, pero siguen alimentando la misma idea, ya que se trata de referirse a los desaparecidos para «la lucha "contra el olvido": recordar para no repetir» ${ }^{45}$. Durante el primer mandato de Carlos Menem no van a tener un verdadero impacto en el conjunto de la sociedad, pero sí lo tendrán en un ámbito particular, privado, que contribuirá ampliamente a uno de los objetivos de las asociaciones: dirigirse a las nuevas generaciones de argentinos.

\section{El SURGimiento De H.I.J.O.S. (1995-2000)}

En abril de 1995, nace una nueva asociación: H.I.J.O.S. (Hijos por la Identidad y la Justicia contra el Olvido y el Silencio), constituida por hijos de desaparecidos, exiliados y presos políticos ${ }^{46}$. Con una edad media de entre 15 y 20 años, sus miembros pueden dirigirse con más facilidad a toda una generación de personas que no conoció ni la dictadura, ni los primeros años de la democracia, jóvenes a los cuales las otras asociaciones de víctimas intentan dirigirse sin mucho éxito. Una generación que se hace adulta durante el primer mandato de Carlos Menem, en medio de una serie de discursos que, como ya hemos evocado, pretenden dejar a un lado el pasado y tienen una circulación importante en los medios de comunicación. Por otra parte, la ausencia prácticamente absoluta de las asociaciones de víctimas en el espacio público argentino, en esta primera mitad de los años 1990, limita la circulación de sus discursos. Esos jóvenes, esos desaparecidos, que «lucharon por un mundo mejor», y cuya lucha «hay que retomar» — no hay que olvidar para que lo que les pasó no vuelva a ocurrir ${ }^{47}$ - , son desconocidos para esta nueva generación.

El nacimiento de H.I.J.O.S. significará un elemento esencial en la evolución de la lucha de estas asociaciones. Pero otro acontecimiento contribuirá

45 Como señala la socióloga Elizabeth Jelin: «El espacio de la memoria es entonces un espacio de lucha política y no pocas veces esta lucha es concebida en términos de la lucha "contra el olvido": recordar para no repetir». Jelin, 2001: 6.

46 Véase: Braun, 2001. Gelman y La Madrid, 1997.

47 Retomamos aquí los principales discursos de las asociaciones que son el objeto de este artículo. 
además a dar una mayor visibilidad a la acción y al discurso de las asociaciones. A principios del año 1995, un exmiembro de la Armada, Adolfo Scilingo, declara en un libro y en televisión haber participado en el asesinato de unas treinta personas ${ }^{48}$ durante la dictadura. Para las asociaciones de víctimas, estas declaraciones son chocantes, en la medida en que presuponen que si Scilingo habló fue porque se sabía protegido por la Ley de Obediencia Debida. Sin embargo, el impacto de las declaraciones de este exmiembro de la Armada en la sociedad argentina es tal que no puede negarse que sus declaraciones, y las de otros miembros de las Fuerzas Armadas a continuación, han contribuido ampliamente al retorno de la lucha de las asociaciones en primera plana ${ }^{49}$. La sociedad civil «descubre», o «redescubre», el horror, sobre todo, una generación de jóvenes que no vivió la dictadura o que era demasiado joven durante el gobierno de Alfonsín.

En paralelo, el nacimiento de H.I.J.O.S. aporta una nueva dimensión al drama de los desaparecidos: el de los huérfanos que ha dejado la dictadura. Por la edad, y sobre todo por la forma de expresión y las acciones, los discursos y declaraciones de este colectivo consiguen llegar a los jóvenes que «descubren» los horrores de la dictadura por medio de las declaraciones de los miembros de las Fuerzas Armadas. La más emblemática de sus acciones es el «escrache» ${ }^{50}$, una iniciativa que exige una preparación de unos tres meses y culmina en una marcha a la vivienda o al lugar de trabajo de un represor identificado y designado como tal. La principal consigna que acompaña los «escraches» es «si no hay justicia, hay escrache» ${ }^{51}$. Se trata de suplir la

48 Verbistky, 1995 (2006). Se refiere sobre todo a los llamados «vuelos de la muerte», durante los cuales, tras inyectarles una dosis de «pentonaval» para adormecerles, se tiraba a personas secuestradas en los centros clandestinos de detención a un río o al mar. En el caso de Scilingo, el centro clandestino era la Escuela de Mecánica de la Armada (ESMA) y se tiraba a las personas al Río de La Plata.

49 Feld, 2002: 103-111.

50 «Escrachar» es un verbo que proviene del lunfardo, lengua coloquial, de la región del Río de La Plata. Tiene varios sentidos que todos tienden a evocar la revelación de algo. La idea es que algo salga a la luz y que, en general, esto sea algo vergonzoso. Para los miembros de H.I.J.O.S. se trataba, en una época en la que no se podían emprender trámites judiciales, de poner en evidencia a personas de diferentes sectores que habían participado de una forma u otra a la represión dictatorial. El objetivo era que estas personas, que vivían en medio del resto de la sociedad argentina, fuesen marcadas e identificadas por sus acciones durante la dictadura. La condena era entonces social, ya que no podía ser judicial.

51 En la actualidad, hay menos «escraches» en la medida en que los miembros de H.I.J.O.S. plantean que las necesidades no son las mismas. Al haberse reiniciado los juicios a partir del año 2005, tras la derogación de las leyes de Punto Final y Obediencia Debida, ya se pueden procesar a los represores de la dictadura. Pero también se limita el uso del «escra- 
ausencia de procesos judiciales gracias a una denuncia «social», que moviliza a los vecinos de represores, o de colaboradores de la dictadura, y pone a estos últimos en evidencia. Huelga decir que esta modalidad aporta un nuevo enfoque a los relatos en torno a la represión dictatorial al centrar el relato en el represor. Mientras las otras asociaciones de víctimas centraron sus discursos y sus acciones esencialmente en los desaparecidos, los hijos de estos ponen a los represores en primer plano. «Cuentan» otro relato, el de los culpables, y ya no el de las víctimas ${ }^{52}$. H.I.J.O.S. permite entonces que las otras asociaciones estén de nuevo muy presentes en el espacio social argentino. Así es cómo, en la conmemoración de los 20 años del golpe de Estado, el 24 de marzo de 1996, todas las asociaciones de familiares y la de sobrevivientes se unieron - con excepción de las MPM - para manifestarse juntas. En esta ocasión, un gran número de personas les apoya, reaccionando a las declaraciones de los exmiembros de las Fuerzas Armadas y a la creación de H.I.J.O.S. ${ }^{53}$.

Lo mismo ocurre cuando, en 1998, Carlos Menem y su gobierno deciden proponer la destrucción de la Escuela de Mecánica de la Armada (ESMA), uno de los centros clandestinos de detención más emblemáticos, y hacer un parque de la memoria. La oposición de las asociaciones de víctimas y de otros sectores es difundida por los medios de comunicación y en la esfera pública ${ }^{54}$. Logran que no se destruya.

Las conmemoraciones en nombre de «la memoria» de los desaparecidos son cada vez más numerosas $\mathrm{y}$, sea cual sea la conmemoración, suele haber un miembro de una de las asociaciones. Las Madres LF, las MPM, las Abuelas y los hijos de desaparecidos están muy presentes en los medios de comunicación, en programas de televisión entre otros, a finales de los años $1990^{55}$. En las escuelas, como en otras instituciones, se solicita cada vez más la presencia de los sobrevivientes para hablar con los niños ${ }^{56}$. Se produce entonces un verdadero revuelo, pero no podemos hablar de reorientación de la lucha.

che», como nos dijo un miembro de H.I.J.O.S. Capital, para evitar que esto lleve a un represor susceptible de ser juzgado o de ser objeto de una querella que se escape. Entrevista del autor con EP, miembro de H.I.J.O.S. Capital, el 22 de diciembre de 2006.

52 Esto se refiere al «escrache», los hijos testimoniaron a menudo sobre sus vivencias en distintos soportes: televisión, películas y libros.

53 Lorenz, 2002: 53-100.

54 Dos fallos para no demoler, Clarín, 31 de enero de 1998, disponible en http://www. clarin.com/diario/1998/01/31/t-01202d.htm.

55 Feld, 2008: 123-136.

56 Entrevista de la autora con $\mathrm{OB}$, miembro de la asociación de exdetenidos-desaparecidos, 15 de diciembre de 2006. 
Como hemos podido observar en entrevistas con distintos miembros de estas asociaciones, todas las asociaciones, las MPM incluidas, destacan la importancia del nacimiento de H.I.J.O.S. ${ }^{57}$. Todos señalan que el nacimiento de esta agrupación ha supuesto un cambio. Sin embargo, esta idea puede matizarse, ya que los miembros de H.I.J.O.S. indican que la creación de su asociación se hizo dentro de una continuidad: «No vinimos a cambiar nada sino a aportar. No empezamos de cero, había todo un camino recorrido durante veinte años y sin ese camino nosotros tampoco hubiésemos podido surgir» ${ }^{58}$.

El primer argumento que viene a la mente para explicar este fenómeno es el lazo de parentesco que condiciona la existencia misma de todas estas asociaciones - a excepción de la de exdetenidos-desaparecidos ${ }^{59}$ - , siendo algunos miembros importantes de la asociación H.I.J.O.S. nietos de Madres LF60. Pero en ese caso, limitaríamos el nacimiento de H.I.J.O.S. a una simple cuestión de influencia. Al ver actuar las otras asociaciones, cuyos miembros eran a veces de sus propias familias, los hijos de desaparecidos habrían decidido crear una asociación. Solo les faltaba tener la edad adecuada para hacerlo. En ese caso, las otras asociaciones habrían sido espectadores del nacimiento de H.I.J.O.S. Pensamos que la relación entre estas asociaciones es un poco más compleja.

En los años 1980 se crearon unos «talleres» en distintos lugares del país. Se trataba de reuniones de hijos de desaparecidos supervisadas por familiares de desaparecidos y sobrevivientes de centros clandestinos de detención. Había una voluntad de relacionar a estos niños entre sí, de mostrarles que no eran los únicos que vivían con la desaparición forzada de sus padres y que otros sufrían situaciones parecidas. Los «talleres» solo existirán durante unos años, pero esta experiencia será la primera iniciativa para reunir a estos hijos en un mismo grupo. No podemos hablar todavía de un colectivo o de una asociación, ya que en este momento los hijos de desaparecidos eran niños en su gran mayoría ${ }^{61}$.

57 Todas las referencias a miembros de las asociaciones objeto de este estudio se refieren a una serie de entrevistas realizadas por la autora entre el 8 de noviembre de 2006 y el 24 de enero de 2007.

58 Madres de Plaza de Mayo-Línea Fundadora, 2006: 174.

59 Acerca de cierta jerarquización en las relaciones entre las asociaciones, véase: Da Silva Catela, 2001.

60 Es el caso de Carlos Pisoni o de Paula Maroni, por ejemplo.

61 Algunos miembros de la asociación eran adolescentes en esta época. Pero eran pocos. Entrevista de Alba Camargo, miembro de H.I.J.O.S. Córdoba, Archivo Memoria Abierta, Buenos Aires. 
La idea de constituir una asociación de hijos de desaparecidos se concretará más tarde, en noviembre de 1994, durante una conmemoración en una universidad de la capital de la provincia de Buenos Aires, La Plata. Gracias a la iniciativa de sobrevivientes de centros clandestinos de detención y de familiares, algunos hijos de desaparecidos que estudiaron en esta universidad antes de «desaparecer» hablan en público ${ }^{62}$. La voluntad de reunir a estos hijos, cuya media de edad se sitúa entre 15 y 20 años, es significativa, cuanto más que ahora pueden hablar en público y evocar sus propias vivencias. A raíz de este primer acontecimiento en el que hijos de desaparecidos llegan a conocerse, estos deciden conformar una agrupación y van a beneficiarse de la ayuda de las asociaciones existentes. Por ejemplo, cuando la asociación H.I.J.O.S. se materializa en la provincia de Buenos Aires, en un principio obtienen ayuda para su organización práctica de las Madres LF, de Familiares, y hasta de las MPM $^{63}$. La relación con las otras asociaciones no se limita entonces a una cuestión de influjo, es mucho más profunda ${ }^{64}$. Estas aseguraron la renovación de su acción, adaptándose a las nuevas generaciones, manteniendo cierta continuidad - a grandes rasgos, los discursos y las reivindicaciones son los mismos para H.I.J.O.S. y para las otras asociaciones-6 ${ }^{65}$. El nacimiento de H.I.J.O.S. contribuyó a situar de nuevo en primer plano las cuestiones ligadas a los desaparecidos y, sobre todo, permitió aportar un nuevo contingente de personas a la lucha contra las llamadas políticas del «perdón» $\mathrm{y}$ en favor de «la memoria», implementada por las asociaciones de víctimas.

El nacimiento de H.I.J.O.S. surge en gran parte gracias a la reorientación de las modalidades de acción de las otras asociaciones de víctimas durante la época en la que los reclamos por la justicia no pueden llevarse a cabo. Se trata de una época en la que las asociaciones de víctimas se vuelcan, de una forma u otra, en una «labor de memoria». La renovación de las modalidades de acción no va sin embargo acompañada de un cambio de discurso, ya que H.I.J.O.S., en sus inicios, se sitúa claramente en la continuidad de las otras asociaciones. No obstante, puede decirse que el «escrache» trae de nuevo al espacio público argentino el reclamo por la justicia, pero que esta vez no pasa

62 Braun, 2001: 21.

63 El primer local de H.I.J.O.S. Capital estaba situado dentro de las dependencias de Familiares, más tarde se alojarían en el local de Madres LF, hasta obtener su propio local. Actualmente han vuelto a la sede de Familiares.

64 Braun, 2001: 28-30.

65 H.I.J.O.S. retomó las consignas históricas de las otras asociaciones y añadió otras que siguen la línea de las asociaciones de víctimas. 
por los tribunales sino por la calle. El concepto de justicia vuelve al escenario público, con las herramientas que aporta H.I.J.O.S., pero transformado y ligado a la lucha contra la impunidad y por «la memoria».

\section{CONCLUSIÓN}

Las asociaciones de víctimas intentaron a lo largo de los años 1990 construir un discurso que contribuyera a la elaboración de una memoria colectiva y que se opusiera a las políticas del «perdón». Al retomar las consignas de las otras asociaciones de víctimas y aportar modalidades de acción propios de su generación, H.I.J.O.S. volvió a dar visibilidad a la cuestión de los desaparecidos. Nuestro objetivo ha sido demostrar que tras el fin de la dictadura en 1983, con el regreso del orden constitucional, la lucha y los discursos de estas asociaciones se orientaron, primero, hacia el reclamo por la justicia y, después, hacia la «labor de memoria». Al distinguir estos dos frentes, hemos querido trasladar la cronología comúnmente utilizada para evocar la evolución del movimiento de derechos humanos argentino a la acción de las asociaciones de víctimas de la dictadura. Se ha tratado de subrayar que, tras la imposibilidad de seguir luchando en la Argentina por el reclamo judicial, estas asociaciones centraron su labor cada vez más en recursos y discursos orientados a la «labor de memoria». Sin embargo, con la llegada de H.I.J.O.S., la relación entre estos dos elementos evoluciona: permitió no solo una mayor difusión del discurso de las asociaciones, sino también el establecimiento del reclamo por la justicia dentro de las reivindicaciones fundamentales de diversos sectores en Argentina.

Este artículo trata de poner de relieve cómo la imposibilidad de llevar a cabo la ausencia de uno de los pilares de la lucha de las asociaciones de víctimas de la dictadura contribuyó ampliamente a desarrollar un reclamo que en los primeros años de la democracia no estaba tan presente: la «labor de memoria». Los años 1980 estuvieron muy marcados por la legitimación del Estado de derecho por vía judicial. Las iniciativas del gobierno de Alfonsín para procurar solucionar las cuestiones ligadas al pasado dictatorial, gracias a instancias como la CONADEP o el juicio a las juntas, asentaron la idea de que la democracia solo podía existir si la justicia tomaba en sus manos la cuestión de los desaparecidos. Por eso, cuando surge la asociación H.I.J.O.S. con su consigna «si no hay justicia, hay escrache», la respuesta por parte de la sociedad civil es inmediata. Por otra parte, por mucho que no se haya podido enjuiciar a los actores de la represión dictatorial, el reclamo judicial nunca desapareció. A partir de la segunda mitad de los años 1990 y principios 
de los 2000, se multiplicaron, con más o menos éxito, las iniciativas internacionales y nacionales para esquivar las leyes de impunidad argentinas ${ }^{66}$.

Todo esto contribuyó a que Néstor Kirchner, durante su presidencia entre 2003 y 2007, tomase en cuenta los reclamos judiciales y memoriales dentro de sus primeras iniciativas como jefe de Estado. La anulación de las leyes de Punto Final y Obediencia Debida en 2005 y de los indultos de Carlos Menem en 2007 permitieron la apertura de casos judiciales en todo el país. La lucha contra la impunidad culminó entonces con la reanudación de los juicios. De nuevo, los tribunales son un escenario válido para las asociaciones de víctimas, las que multiplican su acción para que se juzgue a los represores. No obstante, el fallecimiento de muchos de estos y el envejecimiento de los testigos limitan en el tiempo esta acción. A medida que pasan los años, la lucha judicial depende cada vez más de lo que ocurre en el ámbito de las luchas ligadas a la «labor de memoria». Su aceptación y su impacto en la sociedad están condicionados por los discursos memoriales que circulan por el país. Ahora bien, como señala la socióloga Elizabeth Jelin, hay actualmente muchas memorias en competencia ${ }^{67}$. Si en los años ochenta el reclamo judicial estaba ligado al establecimiento del Estado de derecho y en los años noventa contribuyó a la lucha contra el olvido, podemos preguntarnos: ¿qué huella van a dejar los juicios que se celebren ahora ante la multiplicación de los relatos e interpretaciones en torno a los desaparecidos y la represión dictatorial?

66 No podemos detallar todas las iniciativas, ya que son muy numerosas. En la Argentina, podemos hablar de los «juicios por la verdad», que obligaban a represores, en nombre de la verdad, a relatar los hechos en torno a la desaparición forzada de una persona. Las declaraciones no podían llevar a una inculpación, pero sirvieron para que los familiares supieran qué pasó con los desaparecidos. Tuvieron lugar en las ciudades de La Plata y de Mar del Plata principalmente. También hubo numerosos intentos para declarar la inconstitucionalidad de las leyes de Punto Final y Obediencia Debida, algunos incluso tendrán cierto éxito, ya que se declaró en 1998 la no retroactividad de estas dos leyes. Pero esto no permitirá que se inicien juicios de nuevo. En el extranjero, numerosos países europeos abrieron causas en contra de represores argentinos, el caso más célebre es el del propio Scilingo, condenado a 640 años de prisión en abril de 2005. Para más detalles sobre estas iniciativas, véase la página web del Centro de Estudios Legales y Sociales (CELS): www.cels.org.ar.

67 Jelin, 2001: 127. 


\section{BIBLIOGRAFÍA}

Acuña, Carlos y Smulovitz, Catalina, «Militares en la transición argentina: del gobierno a la subordinación constitucional», Acuña, C. et al., Juicio, castigos y memorias, Buenos Aires, Nueva Visión, 1995.

Braun, Gladis Mabel, Hijos de la ausencia, H.I.J.O.S. para la memoria, Misiones, Maestría en Antropología Social, Universidad Nacional de Misiones bajo la dirección de Leopoldo J. Bartolomé, marzo de 2001, inédito.

CONADEP, Nunca Más, Buenos Aires, Eudeba, 1985.

CONADEP, Nunca Más, Buenos Aires, Eudeba, 2006.

Crenzel, Emilio, La historia política del Nunca Más, Buenos Aires, Siglo XXI Editores, 2008.

Da Silva Catela, Ludmila, No habrá flores en la tumba del pasado, La Plata, Al Margen, 2001.

Feld, Claudia, Del estrado a la pantalla: las imágenes del juicio a los ex comandantes en Argentina, Buenos Aires, Siglo XXI Editores, 2002.

Franco, Marina y Tahir, Nadia, «Associations de victimes, terrorisme d'État et politique dans l'Argentine de 1973 à 1987», Vingtième Siècle. Revue d'Histoire, 105 (París, 2010): 185-198.

Gelman, Juan y La Madrid, Mara, Ni el flaco perdón de Dios. Hijos de desaparecidos, Buenos Aires, Planeta, 1997.

Gonzalez Bombal, Inés, «"Nunca más”, el juicio más allá de los estrados», Acuña, C. et al., Juicio, castigos y memorias, Buenos Aires, Nueva Visión, 1995.

Gorini, Ulises, La rebelión de las Madres, Buenos Aires, Norma, 2006.

Gorini, Ulises, La otra lucha, Buenos Aires, Norma, 2008.

Jelin, Elizabeth, Los trabajos de la memoria, Madrid, Siglo XXI Editores, 2001.

Jelin, Elizabeth (dir.), «Los sentidos de la conmemoración», Las conmemoraciones: las disputas en las fechas in-felices, Buenos Aires, Siglo XXI Editores, 2002: 245-250.

Jelin, Elizabeth, «Los derechos humanos entre el Estado y la sociedad», Suriano, Juan, Nueva historia argentina, dictadura y democracia (1976-2001), Buenos Aires, Editorial Sudamericana, 2005, tomo X.

Lefranc, Sandrine, Politiques du Pardon, París, PUF, 2002.

Leis, Héctor Ricardo, El movimiento por los derechos humanos y la politica argentina /1 y 2, Buenos Aires, Centro Editor de América latina, 1989.

Longoni, Ana, Traiciones, Buenos Aires, Norma, 2007. 
Lorenz, Federico Guillermo, «¿De quién es el 24 de marzo? Las luchas por la memoria del golpe de 1976», Jelin, Elizabeth, Las conmemoraciones: las disputas en la fechas in-felices, Madrid, Siglo XXI Editores, 2002: 53-100.

Madres de Plaza de Mayo-Línea Fundadora, Memoria, verdad y justicia. A los treinta años por los treinta mil, Buenos Aires, Baobab, 2006.

Mellibovsky, Matilde, Círculo de amor sobre la muerte, Buenos Aires, Colihue, 1989 (2006).

Nino, Carlos, Juicio al mal absoluto, Buenos Aires, Ariel, 1997 (2. a edición, 2006).

Nora, Pierre, Les lieux de la Mémoire, vol. 1, La République, París, Quarto Gallimard, 1997.

Novaro, Marcos, Historia de la Argentina contemporánea. De Perón a Kirchner, Buenos Aires, Edhasa, 2006.

Pereyra, Sebastián, ¿La lucha es una sola?, Buenos Aires, Los Polvorines-Univ. Nacional General Sarmiento, Buenos Aires-Biblioteca Nacional, 2008.

Ricoeur, Paul, La mémoire, l’histoire, l'oubli, París, Editions du Seuil, collection Points, 2000.

Tahir, Nadia, «Les associations de victimes de régime autoritaire de 1976-1983 et le pouvoir politique en Argentine», Fregosi, Renée y España, Rodrigo, Droits de l'homme et consolidation démocratique en Amérique du Sud, París, L'Harmattan, 2009: 233-249.

Traverso, Enzo, Le passé, modes d'emploi. Histoire, mémoire, politique, París, La fabrique, 2005.

Verbistky, Horacio, El Vuelo, Buenos Aires, Editorial Sudamericana, 1995 (2006).

Vezzetti, Hugo, Pasado y Presente. Guerra, dictadura y sociedad en la Argentina, Buenos Aires, Siglo XXI editores Argentina, 2003.

\section{MATERIAL DE LAS ASOCIACIONES}

«No al indulto», Revista de Familiares, Decimos, XII/3 (Buenos Aires, 1989): 1.

Llamamiento para participar en Radio Memoria para la semana internacional del detenido-desaparecido, 23 de mayo de 1994.

Editorial, Decimos, Revista de Familiares, II/4 (Buenos Aires, 1998): 1.

Familiares de desaparecidos y detenidos por razones políticas para los 30 años de su existencia en el año 2006: Testimonios de nuestra lucha 1976-2006, Buenos Aires, 2006.

Editorial, Revista de las Madres de Plaza de Mayo, VIII/86 (Buenos Aires, 1992): 1. 
Editorial, «Tenemos una memoria fértil», Revista de las Madres de Plaza de Mayo, XIV/150 (Buenos Aires, 1998): 3.

Folleto «Nuestras consignas», publicado por la Asociación de Madres de Plaza de Mayo (MPM), verano de 2008.

\section{ENTREVISTAS}

Entrevista de la autora con IDV, miembro fundador de Familiares, 21 de diciembre de 2006.

Entrevista de Alba Camargo, miembro de H.I.J.O.S. Córdoba, Archivo Memoria Abierta, Buenos Aires.

Entrevista de la autora con $\mathrm{OB}$, miembro de la asociación de exdetenidos-desaparecidos, 15 de diciembre de 2006.

Entrevista de la autora con AS, Madre de Plaza de Mayo LF, los 22 y 27 de noviembre de 2006.

\section{PÁGINAS EN INTERNET}

Abuelas de Plaza de Mayo: www.abuelas.org.ar.

Convención Internacional sobre la desaparición forzada de personas en la página web del Alto Comisionado por los Derechos Humanos de la Organización de las Naciones Unidas: http://www.ohchr.org/Documents/Publications/FactSheet6Rev3_ sp.pdf.

Centro de Estudios Legales y Sociales (CELS): www.cels.org.ar.

Universidad de las Madres de Plaza de Mayo: www.madres.org/univupmpm/univumpm.asp.

\section{ARTíCULO DE PRENSA}

«Dos fallos para no demoler», Clarín, 31 de enero de 1998, http://www.clarin.com/ diario/1998/01/31/t-01202d.htm.

Fecha de recepción: 1 de junio de 2010

Fecha de aceptación: 7 de junio de 2011 


\section{Justice and memory: the actions of the victim's associations of the dictatorship in Argentina (1983-2000)}

Between 1976 and 1983, Argentina lived through the bloodiest dictatorship in its history. The most emblematic method of repression by the military juntas in power was the people's enforced disappearance. After the end of the dictatorship, the associations of relatives and missing former detainees were involved in the struggle for the "truth, justice and memory" linked to democracy's processing of the dictatorial past. This work intends to return to the speeches and the actions of these groups during the Presidential terms of office of both Raul Alfonsin (1983-1989) and Carlos Menem (1989-1999). We will see how the situation evolved, going from a period in which their claims were in the foreground of the public sphere and the Government's agenda to becoming a time of the so-called «national reconciliation» policies while in opposition. We'll see how the main players focussed on keeping up their claims and to what extent the new situation allowed for the emergence of new players. We will examine how this group of actors used legal tools and memorials to continue their work and emphasize the uniqueness of the Argentine case in the construction of a collective memory.

Key words: Argentina; dictatorial past; associations; justice; memory. 